\title{
Risk of gestational diabetes mellitus in systemic lupus erythematosus pregnancy: a systematic review and meta-analysis
}

\author{
Yuanyuan Dong ${ }^{1,2+}$, Ziwei Dai ${ }^{1,2 \dagger}$, Zhihui Wang ${ }^{1,2}$, Hong Wang ${ }^{1,2}$, Feifei Yuan ${ }^{1,2}$, Ying Zhu ${ }^{3}$, Dongqing Ye ${ }^{1,2^{*}}$ and \\ Bin Wang ${ }^{1,2^{*}}$ (D)
}

\begin{abstract}
Background: It is well established that the risks of insulin resistance and diabetes mellitus are elevated in systemic lupus erythematosus (SLE) patients. However, the relationship between SLE pregnancy and gestational diabetes mellitus (GDM) is still obscure. We perform the present systematic review and meta-analysis to determine the relationship between GDM and SLE pregnancy.

Methods: According to the Preferred Reporting Items for Systematic Reviews and Meta-Analyses (PRISMA) statement, relevant studies were carefully retrieved through PubMed, Cochrane library and Web of Science, China National Knowledge Infrastructure, Wanfang database and China Biology Medicine database from inception till 30 August 2018. GDM risk ratio (RR) of pregnant SLE patients versus controls was calculated to evaluate the association between GDM and SLE. Pooled RRs and 95\% confidence intervals (Cls) were calculated using random effects model by R software.

Results: The literature retrieval identified 339 potential studies in total, and five studies containing 3432 pregnant participants with $248 \mathrm{GDM}$ events were included finally. Pooled analysis found that the risk of GDM were not significant increased in SLE patients compared to controls ( $R R=1.08,95 \% \mathrm{Cl}=0.49$ to $2.41, \mathrm{Z}=0.19$ and $P=0.848$ ). Nevertheless, meta-regression identified that glucocorticoids use and anti-double stranded DNA antibodies positive of SLE patients were positively associated with the risk of GDM.

Conclusions: Our meta-analysis demonstrated that SLE pregnancy may not increase the risk of GDM, but the steroid use during pregnancy was associated with increased risk of GDM. Further large prospective and basic immunologic studies should be implemented for exploring the mechanism underlying glucocorticoids use and GDM.
\end{abstract}

Keywords: Systemic lupus erythematosus, Pregnancy, Glucocorticoids, Gestational diabetes mellitus

\section{Background}

Systemic lupus erythematosus (SLE) is a chronic autoimmune disease predominantly affecting women of reproductive ages, characterized by loss of self-tolerance, production of autoantibodies and deposition of antigenantibody complexes. SLE usually involved multiple organs, like skin, joints, kidneys and nervous system. SLE is reported to involve multiple organs like skin, joints, kidneys and nervous system. Besides, pregnancy

\footnotetext{
* Correspondence: ydq@ahmu.edu.cn; wangbin@ahmu.edu.cn

'Yuanyuan Dong and Ziwei Dai are contributed equally to this work, and they should be viewed as joint first authors

'Department of Epidemiology and Biostatistics, School of Public Health, Anhui Medical University, 81 Meishan Road, Hefei 230032, Anhui, China Full list of author information is available at the end of the article
}

is also dangerous for women with SLE. Recently, more studies focus on the higher risks of pregnancy complications in SLE patients, especially in patients with the existence of autoantibody positive, antiphospholipid syndrome (APS), nephritis or pulmonary hypertension, and patients with immunosuppressive therapies [1]. Although the fertility rate of SLE women is normal, SLE pregnancy was reported to significant increased risk of adverse maternal and fetal adverse outcome [2]. Increasing numbers of studies reported that autoimmune diseases including SLE could affect the process of pregnancy and induce adverse pregnancy outcomes (APOs), such as gestational hypertension, pre-eclampsia and lupus flares [3-6]. Gestational diabetes mellitus (GDM)

(c) The Author(s). 2019 Open Access This article is distributed under the terms of the Creative Commons Attribution 4.0 International License (http://creativecommons.org/licenses/by/4.0/), which permits unrestricted use, distribution, and reproduction in any medium, provided you give appropriate credit to the original author(s) and the source, provide a link to the Creative Commons license, and indicate if changes were made. The Creative Commons Public Domain Dedication waiver (http://creativecommons.org/publicdomain/zero/1.0/) applies to the data made available in this article, unless otherwise stated. 
is the most common autoimmune endocrine complication of pregnancy. However, the relationship between GDM and SLE has not been well illustrated yet.

GDM is a common pregnancy complication, defined as impaired glucose tolerance first detected during pregnancy, and is associated with adverse maternal and fetal outcomes, including hyperinsulinemia, hypocalcemia, hyperbilirubinemia, preeclampsia, and macrosomia $[7,8]$. The risk of dystocia, usually caused by macrosomia, cesarean section and even stillbirth are also increased in pregnancy women with GDM $[9,10]$. For the long-dated consequences, GDM is related to markedly increased risk of post-partum diabetes and cardiovascular diseases $[7,11]$. Although the exact pathological mechanism underlying GDM is still obscure, exacerbated insulin resistance is reported to play a pivotal role in GDM. SLE is also reported to associate with increased risk of insulin resistance and diabetes mellitus. Besides, studies also indicated that abnormal insulin secretion may be caused by autoimmune damage [12], and autoimmune response may impair the function of pancreatic beta cell [13]. Patients with SLE are accompanied with autoimmune microenvironment during pregnancy, which may relate to GDM incidence. Present studies indicated that SLE pregnancy is associated with elevated risk of GDM [10]. However, studies also reported that the GDM risk of SLE patients was not significant increased [9, 14, 15]. Besides, whether there are specific risk factors influencing GDM incidence in SLE patients is also doubtful. In light of these facts, we performed this study to comprehensively review and meta-analyze the relationship between SLE and GDM.

\section{Methods}

This study was performed was conducted according to the Preferred Reporting Items for Systematic Reviews and Meta-Analyses (PRISMA) statement [16] (see Additional file 1: Table S1) .

\section{Review question}

According to the PICOs scheme, the review question of our meta-analysis was whether pregnant women with SLE (Participants) were associated with increased risk ratio (RR) of GDM (Outcomes) compared with non-SLE pregnant women (Comparisons) of cohort and case-control studies (Study designs).

\section{Search strategy and study selection}

PubMed, Web of Science, and Cochrane Library were comprehensive searched for relevant studies from inception to 30 August, 2018. MeSH terms and Web of Knowledge topics were used. The detailed search strategy was provided in Additional file 1: Figure S1 To identify the potential grey literatures, we also searched relevant studies of our analysis in three Chinese databases, China National
Knowledge Infrastructure, Wanfang database and China Biology Medicine database. Furthermore, bibliographies from eligible original studies and reviews were also searched manually.

The two reviewers (Yuanyuan Dong, Ziwei Dai) firstly performed an initially title and abstract screening independently. Studies fully agreed on eligible for inclusion by two reviewers will be further assessed for availability through full-test review. Eligible studies must respectively fulfill the inclusion criteria as following: a) article reported the incidence risk of GDM in SLE pregnant women compared with controls; b) article reported original data eligible for calculate of the index; c) SLE was diagnosed according to ACR criteria. d) GDM was defined as any degree of glucose intolerance with onset or first detected during pregnancy or diagnosed as included studies reported criteria [17]; e) original research designed as case-control or cohort study. When necessary, we contacted corresponding authors for full-text or relevant data. Additionally, the studies in the forms of case reports, clinical trials and reviews were excluded. There was no language restriction. To avoid including data of duplicate publication, only most comprehensive study including more abundant data was analyzed.

\section{Methodological quality assessment and data extraction}

Data extraction and methodological assessment were conducted by two investigators (Yuanyuan Dong, Ziwei Dai) independently and confirmed by a third reviewer (Zhihui Wang). The Newcastle-Ottawa Scale (NOS) for cohort and case-control studies were used to assess the quality [18]. For further exploring the impact of SLE on GDM risk, the following characteristics were also extracted from included studies: publication year, first author's name, region, disease duration, diagnosis criteria of GDM and SLE, study design, sample size, age of pregnant women of SLE patients and controls. In addition, some clinical characteristics of SLE, like proportions of antiphospholipid antibody (aPL) positive, anti-double stranded DNA antibodies (anti-ds-DNA) positive, lupus nephritis, and hydroxychloroquine or glucocorticoids use () were also obtained. Any discrepancy in processes of literature search, study selection, quality assessment or original data extraction was resolved with consensus via discussing.

\section{Statistical analysis}

Original data regarding GDM risk of SLE patients compared to controls was used to calculate the effect size. The relationship between SLE and GDM was evaluated by RR and 95\% confident interval (CI) according to either fix effect model or randomized effects model, depending on the heterogeneity among included studies. Cochran's Q statistic was used to assess between studies heterogeneity; besides, $I^{2}$ test was also used to quantify the degree of inconsistency by calculating the percentage 
of total variation between studies, where due to heterogeneity rather than chance. Heterogeneity graded by $I^{2}$ was set as low $(<25 \%)$, medium (25-75\%) and high ( $\geq 75 \%)$ [19]. If there was a significant heterogeneity, the random effects model is chosen, otherwise the fix effect model is chosen $[20,21]$, visualized by forest plots. Sensitivity analysis was performed to validate the stability of the meta-analysis by consecutively excluding each enrolled study. Any $P<0.05$ was considered as statistically significant, and all statistical analyses were conducted by $\mathrm{R}$ software ( $\mathrm{R}$ Foundation for Statistical Computing, Vienna, Austria).

\section{Results}

\section{Search results}

This search identified 339 references totally from PubMed $(n=182)$, Web of Science $(n=83)$, Cochrane Library $(n=$ 7), China National Knowledge Infrastructure $(n=1)$, Wanfang database $(n=38)$ and China Biology Medicine database $(n=28)$ (Fig.1). After removal of duplicates and screening of title and abstract, a total of 23 records were obtained for full-text review. No any additional study was retrieved from references search. At last, five studies containing 714 SLE patients and 2718 controls were eligible for inclusion. In addition, the NOS scores of all included studies were acceptable detailed in Table 1. Nevertheless, methodological flaws were still existent. First, the sample sizes of some studies were small $[15,22]$. The diagnosis criteria for APOs including GDM were obscure [14, 15, 22]. Besides, factors as previous pregnancy history of SLE patients and controls were not well controlled.

\section{Characteristics of studies}

The baseline characteristics of five eligible studies $[9,10$, 14, 15, 22] were listed in Table 1 and Additional file 1: Table S2 Except for study by Yan Yuen [22], included studies were all conducted in Asia. Four studies reported that SLE was diagnosed according to ACR criteria, and study by Phansenee S et al. has not reported SLE diagnostic criteria [14]. Two studies have not indicated the disease duration of SLE patients [10, 14]. All the included studies were retrospectively designed. Two studies were case-control designed $[15,22]$ and three studies were cohort designed $[9,10,14]$.

$\mathrm{Wu} J$ et al. reported significantly decreased risk of GDM in SLE patients than controls (5.6\% vs $11.5 \%$ ) [9], whereas research by Abdwani $R$ et al. [10] reported that the GDM risk of SLE patients were increased. The remaining three articles have not found any significant difference of GDM risk between SLE patients and controls $[14,15,22]$. Four studies reported the proportions

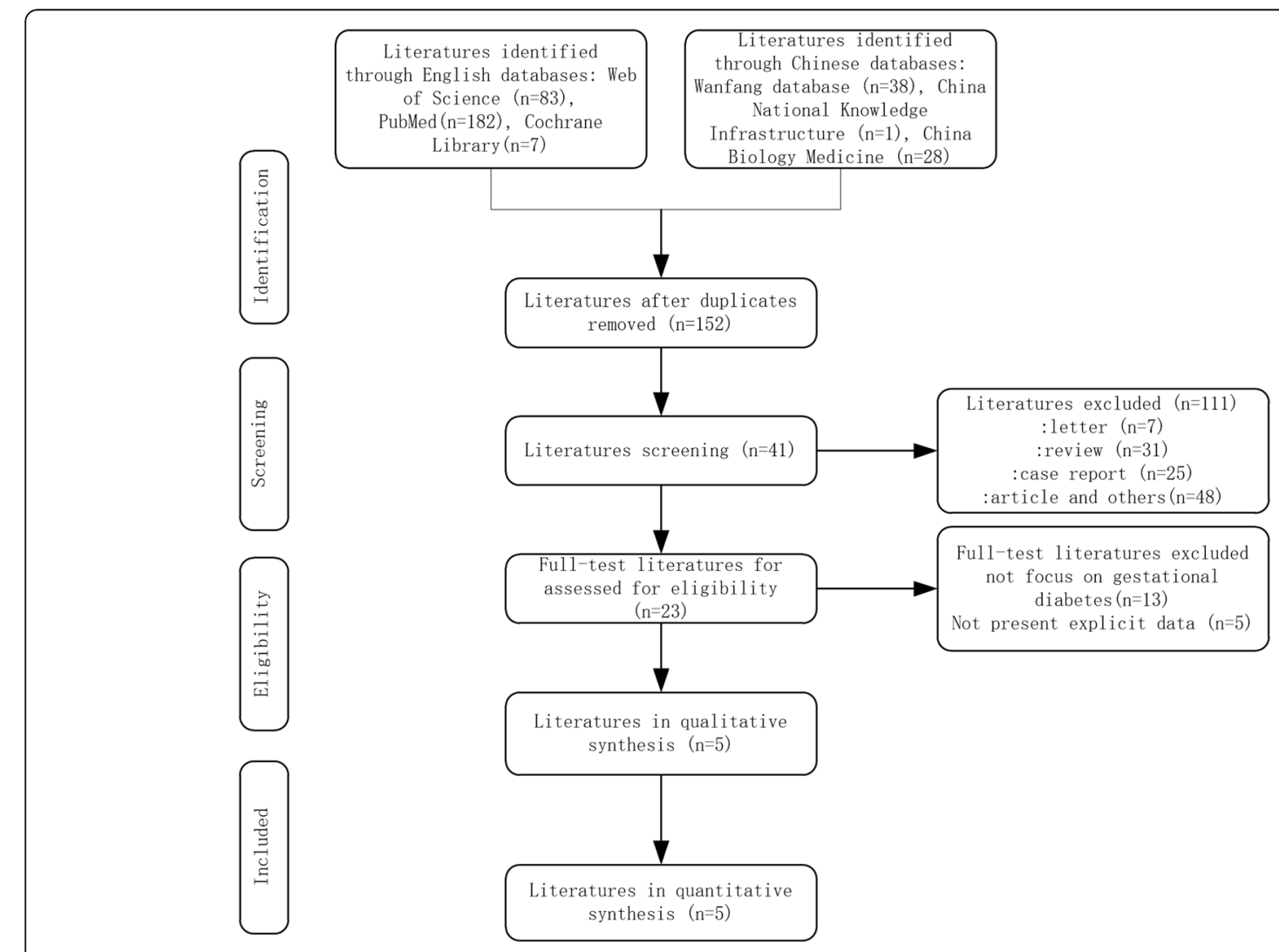

Fig. 1 Flow chart of the literature search and study selection 
Table 1 Characteristics of included studies

\begin{tabular}{|c|c|c|c|c|c|c|c|c|c|c|}
\hline Author & Country & Year & $\mathrm{N}$ of cases & $\mathrm{N}$ of controls & $\begin{array}{l}\text { Average age } \\
\text { of cases }{ }^{\mathrm{a}}\end{array}$ & $\begin{array}{l}\text { Average age } \\
\text { of controls }\end{array}$ & $\begin{array}{l}\text { Disease } \\
\text { duration }^{\text {a }}\end{array}$ & Study type & $\begin{array}{l}\text { Diagnosis } \\
\text { criteria for } \\
\text { SLE }\end{array}$ & $\begin{array}{l}\text { Results of } \\
\text { quality } \\
\text { assessment }\end{array}$ \\
\hline Wu J [9] & China(A) & 2018 & 338 & 1014 & 29.5 & 29.7 & 5.6 & Retro-cohort & $A C R$ & 8 \\
\hline Abdwani R [10] & $\operatorname{Oman}(A)$ & 2018 & 56 & 91 & 31.0 & 29.0 & NA & Retro-cohort & $A C R$ & 8 \\
\hline Phansenee S [14] & Thailand (A) & 2017 & 133 & 1394 & 29.5 & 27.4 & NA & Retro-cohort & NA & 7 \\
\hline Galappatthy P [15] & Sri Lanka (A) & 2017 & 79 & 85 & 25.9 & NA & 8.0 & $\begin{array}{l}\text { Retro-case- } \\
\text { control }\end{array}$ & $A C R$ & 7 \\
\hline Yan Yuen [22] & Canada & 2008 & 108 & 134 & 42.0 & 38.0 & 15.0 & $\begin{array}{l}\text { Retro-case- } \\
\text { control }\end{array}$ & $A C R$ & 8 \\
\hline
\end{tabular}

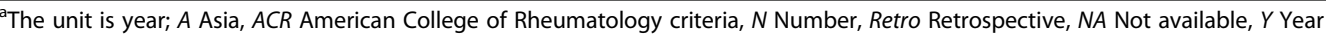

of patients used hydroxychloroquine and glucocorticoids during pregnancy $[9,10,14,15]$. Notably, in research of Wu J et al. [9], about $97.6 \%$ of SLE patients used glucocorticoids during pregnancy.

\section{RR of GDM in SLE pregnancy}

In total, five studies containing 714 SLE pregnant women reported the RR of GDM between 5 and $28.3 \%$. Meta-analysis combining the five studies yield that the pooled RR of GDM was 1.08 (95\% CI $=0.49$ to $2.41, \mathrm{Z}=$ 0.19 and $P=0.848)$ in SLE patients with significant heterogeneity $\left(I^{2}=76 \%\right.$; $\left.\mathrm{Tau}^{2}=0.56, P<0.01\right)$ (see Fig. 2). Metaregression on factors of sample size, age of cases, publication year, and proportions of aPL positive, nephritis and hydroxychloroquine use indicating that none of these factors was the source of heterogeneity. However, glucocorticoids use $(\mathrm{r}=0.0024, P=0.002)$ and anti-ds-DNA positive $(\mathrm{r}=0.0022, P=0.003)$ of SLE patients were reported to positively associate with GDM risk (see Table 2). Sensitivity analysis indicated that the result of meta-analysis was stable whatever which study was omitted (Additional file 1: Figure S2). Because we have five included studies, the evaluation of publication bias was not necessary.

\section{Discussion}

Women with SLE are receiving better multidisciplinary antenatal care ensuring better pregnancy outcome with the development of medicine. Nevertheless, current studies [5, 23-25] also reported that SLE was associated with APOs including pre-eclampsia, pregnancy-induced hypertension, spontaneous abortion. The present study have synthesized current published studies regarding GDM and SLE, and the results indicated that SLE with not associated with GDM. The potential reasons for previous inconsistent results are listed as following.

With abnormal insulin resistance playing an irreplaceable role, GDM was reported to associate with clinical factors in SLE patients. A previous meta-analysis [24] regarding the risk of maternal and fetal outcomes in SLE pregnancy identified that several factors included lupus nephritis [26, 27], aPL positive and APS [28] were responsible for the higher risk of APOs following pregnancy. We also conducted meta-regression analysis on corresponding factors to assess their impact on GDM risk. As previous studies reported that diabetes mellitus was developed around $12 \%$ of SLE patients due to highdose glucocorticoid therapy (prednisolone use $\geq 1 \mathrm{mg} / \mathrm{kg}$ / day) $[29,30]$. Our study also identified that glucocorticoid use during pregnancy was positively associated GDM risk in SLE patients. Consistent with our results, studies also reported that women with higher dose of steroid during pregnancy have an increased risk of diabetes, and steroid exposure should be restricted to a minimum during pregnancy $[4,31]$. Glucocorticoid was

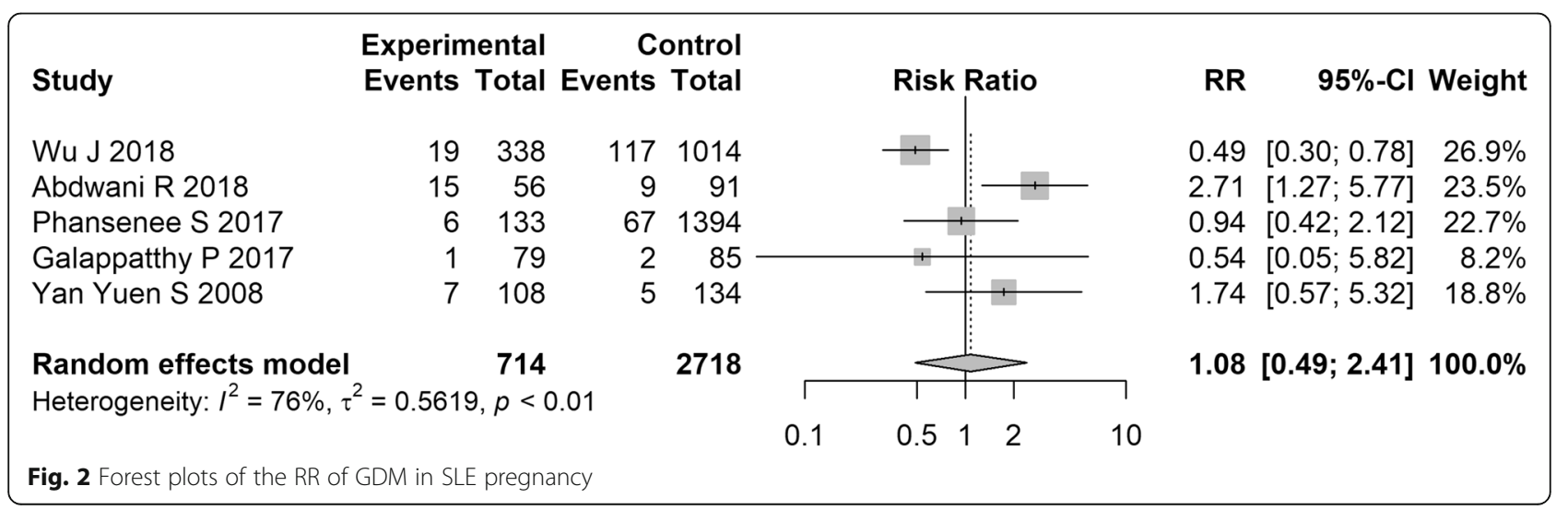


Table 2 Meta-regression analysis coefficients of risk ratio of GDM

\begin{tabular}{|c|c|c|c|c|}
\hline Variables & Coefficient (SE) & $95 \% \mathrm{Cl}$ & $Z$ & $P$ \\
\hline Publication year & $-0.0028(0.0108)$ & {$[-0.0238,0.0183]$} & -0.2557 & 0.800 \\
\hline $\mathrm{N}$ of cases & $0.0004(0.0002)$ & {$[<-0.0000,0.0008]$} & 1.8972 & 0.058 \\
\hline Age of cases & $0.0034(0.0078)$ & {$[-0.0120,0.0187]$} & 0.4334 & 0.665 \\
\hline $\mathrm{aPL}$ & $-0.0066(0.0063)$ & {$[-0.0190,0.0057]$} & -1.0529 & 0.292 \\
\hline anti-ds-DNA & $0.0022(0.0007)$ & {$[0.0008,0.0037]$} & 3.0053 & $0.003^{*}$ \\
\hline Lupus nephritis & $-0.0023(0.0044)$ & {$[-0.0109,0.0064]$} & -0.5146 & 0.607 \\
\hline HCQ use & $0.0021(0.0011)$ & {$[<-0.0000,0.0043]$} & 1.9213 & 0.055 \\
\hline Glucocorticoids use & $0.0024(0.0008)$ & {$[0.0009,0.0040]$} & 3.0512 & $0.002^{*}$ \\
\hline
\end{tabular}

aPL, antiphospholipid antibodies; anti-ds-DNA, anti-double stranded DNA antibodies; HCQ, hydroxychloroquine; Cl, Confident interval; N, Number; SE, Standard error; ${ }^{*}, P<0.05$

known to necessary for the control of SLE disease activity during pregnancy. One original study reported that the higher risk of GDM in SLE patients compared with controls may due to high rate of glucocorticoids use of SLE patients during pregnancy [10]. Our results also demonstrated that hydroxychloroquine use and the number of cases were borderline significant factors positively associated with the GDM risk. However, hydroxychloroquine use may reduce glucocorticoid doses during pregnancy, consequently reducing the risk of GDM development. Studies also reported that hydroxychloroquine is safe enough to continue throughout the whole pregnancy process by all pregnant women with SLE [24, 32]. Simultaneously, epidemiologic studies have demonstrated that hydroxychloroquine could significantly reduce diabetes mellitus risk of SLE patients in a dosedependent manner $[33,34]$. So we hypothesized that the increased risk of GDM by hydroxychloroquine use was a false positive result. Hydroxychloroquine use during SLE pregnancy could be recommended for reducing glucocorticoid doses. The potential reason may be that medication use demands of SLE patients were differential across studies populations depending on disease activity. The hydroxychloroquine use rate was associated with glucocorticoids use rate in some extent. Besides, the glucocorticoids use rate of SLE patients during pregnancy was extremely high. The false positive result may due to the confounding of glucocorticoids. We also found that SLE patients with anti-ds-DNA were associated with higher risk of GDM. Whether it is the consequences of autoimmune dysfunction contributing to autoimmune GDM is still obscure. In words, we hypothesized that the use of glucocorticoids may increase the risk of GDM and the autoimmune dysfunction of SLE may related to autoimmune GDM.

Another reason accounting for the inconsistent results of original studies may be related to the inconsistent diagnosis criteria of GDM. As the most common metabolic disturbance among pregnant women, GDM has a series of diagnosis criteria [32, 33]. A recent meta-analysis by Behboudi-Gandevani $\mathrm{S}$ and colleagues reported that the worldwide prevalence of GDM was $4.4 \%$, regardless of type of screening threshold categories. According to seven different diagnosis criteria, subgroup analysis of the study indicated that the prevalence of GDM was ranged from 2.2 to $10.6 \%$ [29]. The diagnosis criteria of GDM in original studies were different, because of the lack of international consistently diagnosis criteria for GDM. GDM risk of SLE patients is not the primary outcome of most included studies, so the screening methods of GDM have not been exactly reported. In our included studies, only two studies reported that GDM was defined as any degree of glucose intolerance with onset or first recognition during pregnancy $[9,10]$. It is hard for us to evaluate the impact of criteria on GDM risk with the present available data. The inconsistent diagnosis criteria of GDM in original studies have indeed made some certain effects on the final diagnosis of GDM, which may partly account to the significant heterogeneity of our study.

Autoimmune GDM is a subset of GDM with the representation of various autoimmune antibodies (GADA, IA2A, IAA, ZnT8-A), and account for about $10 \%$ of all GDM [13]. Autoimmune GDM was also reported to associate with higher risk of type 1 diabetes or latent autoimmune diabetes in adult. Therefore patients with autoimmune GDM was worthy of studying for the prevention of type 1 diabetes in pregnancy or afterwards [30]. SLE is an autoimmune dysfunction disease and characterizing with the presence of various antibodies, likely to associate with autoimmune GDM. Regrettably, of studies, none of the present studies, researching the relationship between SLE and GDM, reported the results of autoimmune GDM or related antibodies. Further studies focus on the relationship between autoimmune GDM and SLE are also necessary.

The major strength of this present study is that we have integrated existing research using systematic quantitative methods, minimizing the selection and reporting biases. There are also some limitations existing. First, the small sample sizes of our included studies may limit 
the power to find positive association between SLE and GDM. Second, data about demographic characteristics (as body mass index and dietary characteristics) and clinical manifestations (as disease activity and drug usage), associated with GDM risk, was absent in our included studies, and differences of these characteristics across our original studies may account for the significant heterogeneity. Also, screening methods of GDM in our original studies were obscure, which may cause the results varying. Lastly, the significant heterogeneity may limit the generalizability of the pooled results.

\section{Conclusions}

In summary, this meta-analysis suggested SLE is not associated with the risk of GDM and glucocorticoids therapy is associated with the increased risk of GDM in SLE patients. A right time for pregnancy (remission or inactive disease status) and detailed preconception counselling are pivotal for a successful pregnancy. Furthermore, further elaborate prospective cohort studies and immunologic researches are needed to reveal the mechanism underlying GDM and glucocorticoids use.

\section{Additional file}

Additional file 1: Table S1. PRISMA checklist. Table S2. Characteristic about SLE patients of included studies. Figure S1. Search strategy of the electrical databases. Figure S2. Sensitivity analysis of meta-analysis (DOCX $512 \mathrm{~kb})$

\section{Abbreviations \\ ACR: American College of Rheumatology; anti-ds-DNA: Anti-double stranded DNA antibodies; aPL: Antiphospholipid antibody; APOs: Adverse pregnancy outcomes; APS: Antiphospholipid syndrome; Cl: Confidence interval; GDM: Gestational diabetes mellitus; MOOSE: Meta-analysis of observational studies in epidemiology; NOS: Newcastle-Ottawa Scale; PRISMA: Preferred Reporting Items for Systematic Reviews and Meta-Analyses; RR: Risk ratios; SLE: Systemic lupus erythematosus}

\section{Acknowledgements}

We really appreciate the efforts of all the researchers whose articles were included in this study.

\section{Funding}

This is supported by National Natural Science Foundation of China (grant numbers: 81573217, 81172764). The funding body had no role in study design, data collection and analysis, decision to publish, or preparation of the manuscript.

\section{Availability of data and materials}

All data pertaining to this study are included in this published article and its supplementary information files.

\section{Supplementary data}

Supplementary data are available online.

\section{Disclosure statement}

None

\section{Authors' contribution}

YD, ZD, ZW, HW, FY, YZ, DY and BW all involved in processes of study

design, selection of articles, data extraction, statistical analysis and manuscript writing. All authors read and approved the final version of the manuscript.

Ethics approval and consent to participate

Not applicable

Consent for publication

Not applicable

\section{Competing interests}

The authors declare that they have no competing interests.

\section{Publisher's Note}

Springer Nature remains neutral with regard to jurisdictional claims in published maps and institutional affiliations.

\section{Author details}

${ }^{1}$ Department of Epidemiology and Biostatistics, School of Public Health, Anhui Medical University, 81 Meishan Road, Hefei 230032, Anhui, China. ${ }^{2} T$ The Key Laboratory of Major Autoimmune Diseases, Anhui Medical University, 81 Meishan Road, Hefei 230032, Anhui, China. ${ }^{3}$ Department of Obstetrics and Gynecology, the First Affiliated Hospital of Anhui Medical University, Hefei 230032, Anhui, China.

Received: 13 November 2018 Accepted: 3 May 2019

Published online: 22 May 2019

\section{References}

1. Nahal SK, Selmi C, Gershwin ME. Safety issues and recommendations for successful pregnancy outcome in systemic lupus erythematosus. J Autoimmun. 2018:93:16-23.

2. Meyer O. Making pregnancy safer for patients with lupus. Joint, bone, spine. Rev Rhum. 2004;71:178-82.

3. Jakobsen IM, Helmig RB, Stengaard-Pedersen K. Maternal and foetal outcomes in pregnant systemic lupus erythematosus patients: an incident cohort from a stable referral population followed during 1990-2010. Scand J Rheumatol. 2015:44:377-84.

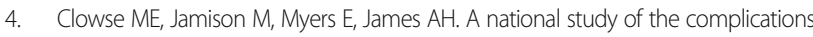
of lupus in pregnancy. Am J Obstet Gynecol. 2008;199:127.e121-6.

5. Smyth A, Oliveira GH, Lahr BD, Bailey KR, Norby SM, Garovic VD. A systematic review and meta-analysis of pregnancy outcomes in patients with systemic lupus erythematosus and lupus nephritis. Clin J Am Soc Nephrol. 2010;5:2060-8.

6. Lateef A, Petri M. Managing lupus patients during pregnancy. Best Pract Res Clin Rheumatol. 2013;27:435-47.

7. Coustan DR. Gestational diabetes mellitus. Clin Chem. 2013;59:1310-21.

8. Kc K, Shakya S, Zhang H. Gestational diabetes mellitus and macrosomia: a literature review. Ann Nutr Metab. 2015:66(Suppl 2):14-20.

9. Wu J, Ma J, Bao C, Di W, Zhang WH. Pregnancy outcomes among Chinese women with and without systemic lupus erythematosus: a retrospective cohort study. BMJ Open. 2018;8:e020909.

10. Abdwani R, Al Shaqsi L, Al-Zakwani I. Neonatal and obstetrical outcomes of pregnancies in systemic lupus erythematosus. Oman Med J. 2018;33:15-21.

11. Ogurtsova $K$, da Rocha Fernandes JD, Huang Y, Linnenkamp U, Guariguata L, Cho NH, Cavan D, Shaw JE, Makaroff LE. IDF diabetes atlas: global estimates for the prevalence of diabetes for 2015 and 2040. Diabetes Res Clin Pract. 2017:128:40-50

12. Lapolla A, Dalfra MG, Fedele D. Diabetes related autoimmunity in gestational diabetes mellitus: is it important? Nutrition, metabolism, and cardiovascular diseases. NMCD. 2009;19:674-82.

13. Cossu E, Incani M, Pani MG, Gattu G, Serafini C, Strazzera A, Bertoccini L, Cimini FA, Barchetta I, Cavallo MG, et al. Presence of diabetes-specific autoimmunity in women with gestational diabetes mellitus (GDM) predicts impaired glucose regulation at follow-up. J Endocrinol Investig. 2018;41:1061-8.

14. Phansenee $S$, Sekararithi $R$, Jatavan $P$, Tongsong T. Pregnancy outcomes among women with systemic lupus erythematosus: a retrospective cohort study from Thailand. Lupus. 2018;27:158-64.

15. Galappatthy $P$, Jayasinghe JDD, Paththinige SC, Sheriff RMH, Wijayaratne LS. Pregnancy outcomes and contraceptive use in patients with systemic lupus erythematosus, rheumatoid arthritis and women without a chronic illness: a comparative study. Int J Rheum Dis. 2017;20:746-54. 
16. Moher D, Liberati A, Tetzlaff J, Altman DG. Preferred reporting items for systematic reviews and meta-analyses: the PRISMA statement. Int J Surg. 2010;8:336-41.

17. Danyliv A, Gillespie P, O'Neill C, Noctor E, O'Dea A, Tierney M, McGuire B, Glynn LG, Dunne F. Short- and long-term effects of gestational diabetes mellitus on healthcare cost: a cross-sectional comparative study in the ATLANTIC DIP cohort. Diabet Med. 2015;32:467-76.

18. Stang A. Critical evaluation of the Newcastle-Ottawa scale for the assessment of the quality of nonrandomized studies in meta-analyses. Eur J Epidemiol. 2010;25:603-5.

19. Higgins JP, Thompson SG. Quantifying heterogeneity in a meta-analysis. Stat Med. 2002;21:1539-58.

20. Higgins JP, Thompson SG, Deeks JJ, Altman DG. Measuring inconsistency in meta-analyses. BMJ (Clin Res Ed). 2003;327:557-60.

21. Borenstein M, Hedges LV, Higgins JP, Rothstein HR. A basic introduction to fixed-effect and random-effects models for meta-analysis. Res Synth Methods. 2010;1:97-111.

22. Yan Yuen S, Krizova A, Ouimet JM, Pope JE. Pregnancy outcome in systemic lupus erythematosus (SLE) is improving: results from a case control study and literature review. Open Rheumatol J. 2008;2:89-98.

23. Wu J, Ma J, Zhang WH, Di W. Management and outcomes of pregnancy with or without lupus nephritis: a systematic review and meta-analysis. Ther Clin Risk Manag. 2018;14:885-901.

24. Bundhun PK, Soogund MZ, Huang F. Impact of systemic lupus erythematosus on maternal and fetal outcomes following pregnancy: a meta-analysis of studies published between years 2001-2016. J Autoimmun. 2017:79:17-27.

25. Wei S, Lai K, Yang Z, Zeng K. Systemic lupus erythematosus and risk of preterm birth: a systematic review and meta-analysis of observational studies. Lupus. 2017;26:563-71.

26. Lin $P$, Rhew E, Ness RB, Peaceman A, Dyer A, McPherson D, Kondos GT, Edmundowicz D, Sutton-Tyrrell $K$, Thompson T, et al. Adverse pregnancy outcomes and subsequent risk of cardiovascular disease in women with systemic lupus erythematosus. Lupus Sci Med. 2014;1:e000024.

27. Yang H, Liu H, Xu D, Zhao L, Wang Q, Leng X, Zheng W, Zhang F, Tang F, Zhang X. Pregnancy-related systemic lupus erythematosus: clinical features, outcome and risk factors of disease flares--a case control study. PLoS One. 2014;9:e104375

28. Lazzaroni MG, Dall'Ara F, Fredi M, Nalli C, Reggia R, Lojacono A, Ramazzotto F, Zatti S, Andreoli L, Tincani A. A comprehensive review of the clinical approach to pregnancy and systemic lupus erythematosus. J Autoimmun. 2016;74:106-17.

29. Behboudi-Gandevani S, Amiri M, Bidhendi Yarandi R, Ramezani Tehrani F. The impact of diagnostic criteria for gestational diabetes on its prevalence: a systematic review and meta-analysis. Diabetol Metab Syndr. 2019:11:11.

30. Haller-Kikkatalo K, Uibo R. Clinical recommendations for the use of islet cell autoantibodies to distinguish autoimmune and non-autoimmune gestational diabetes. Clin Rev Allergy Immunol. 2016;50:23-33.

31. Brucato A, Doria A, Frassi M, Castellino G, Franceschini F, Faden D, Pison MP, Solerte L, Muscara M, Lojacono A, et al. Pregnancy outcome in 100 women with autoimmune diseases and anti-Ro/SSA antibodies: a prospective controlled study. Lupus. 2002;11:716-21.

32. Bitencourt N, Bermas BL. Pharmacological Approach to Managing Childhood-Onset Systemic Lupus Erythematosus During Conception, Pregnancy and Breastfeeding. Paediatr Drugs. 2018;20:511-21.

33. Chen YM, Lin CH, Lan TH, Chen HH, Chang SN, Chen YH, Wang JS, Hung WT, Lan JL, Chen DY. Hydroxychloroquine reduces risk of incident diabetes mellitus in lupus patients in a dose-dependent manner: a population-based cohort study. Rheumatology (Oxford, England). 2015;54:1244-9.

34. Shaharir SS, Gafor AH, Said MS, Kong NC. Steroid-induced diabetes mellitus in systemic lupus erythematosus patients: analysis from a Malaysian multiethnic lupus cohort. Int J Rheum Dis. 2015:18:541-7.

Ready to submit your research? Choose BMC and benefit from:

- fast, convenient online submission

- thorough peer review by experienced researchers in your field

- rapid publication on acceptance

- support for research data, including large and complex data types

- gold Open Access which fosters wider collaboration and increased citations

- maximum visibility for your research: over $100 \mathrm{M}$ website views per year

At $\mathrm{BMC}$, research is always in progress.

Learn more biomedcentral.com/submissions 